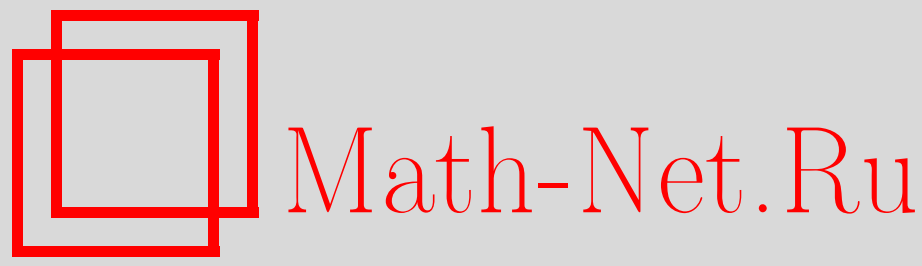

Д. В. Талалаев, Квантовая обобщенная система Тоды, ТМФ, 2012, том 171, номер 2, 312-320

DOI: https://doi.org/10.4213/tmf6902

Использование Общероссийского математического портала Math-Net.Ru подразумевает, что вы прочитали и согласны с пользовательским соглашением http://www . mathnet.ru/rus/agreement

Параметры загрузки:

IP: 35.173 .137 .237

26 апреля 2023 г., $17: 36: 18$

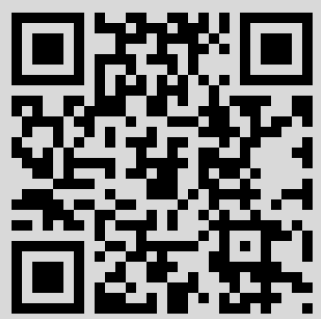


Том 171, № 2

май, 2012

(C) 2012 г. Д. В. Талалаев ${ }^{* \dagger}$

\title{
КВАНТОВАЯ ОБОБЩЕННАЯ СИСТЕМА ТОДЫ
}

\begin{abstract}
Строится "спектральная кривая" для обобщенной системы Тоды, что позволяет эффективно получать квантование данной системы. В свою очередь квантование реализуется с помощью метода квантового характеристического полинома для системы Годена и подходящей редукции Адлера-Костанта-Саймса. Обсуждается также связь полученного результата с недавними рассмотрениями заставы Дринфельда пространств монополей и соответствующих симметрий борелевской версии янгиана.
\end{abstract}

Ключевые слова: квантование, интегрируемые системы, пространство флагов.

К 60-летию И.М. Кричевера

\section{1. ВВЕДЕНИЕ}

Цепочки Тоды занимают особое место в теории интегрируемых систем. В отличие от структуры линейной группы в модели Годена эта система связана с соответствующей борелевской группой и опосредованно с геометрией многообразий флагов.

Предметом изучения в настоящей работе является обобщенная система Тоды, связанная системой корней $A_{n}$. Используемый в работе метод основан на концепции спектральной кривой как на классическом, так и на квантовом уровне. Метод спектральной кривой и вообще алгебро-геометрические методы в интегрируемых системах предоставляют удивительно эффективный и универсальный способ описания, решения и квантования динамических систем. Настоящая работа была инициирована построением коммутативного семейства [1], которое на первый взгляд не связано с идеей спектральных инвариантов для эволюционирующего линейного оператора.

Следуя работе [2], напомним построение спектральной кривой в открытой цепочке Тоды. Открытая цепочка Тоды определяется гамильтоновой функцией

$$
H=\sum_{k=1}^{n} \frac{p_{k}^{2}}{2}+\sum_{k=1}^{n-1} e^{q_{k}-q_{k+1}}
$$

* Московский государственный университет им. М. В. Ломоносова, Москва, Россия

${ }^{\dagger}$ Институт теоретической и экспериментальной физики, Москва, Россия. E-mail: dtalalaev@yandex.ru 
и канонической скобокой Пуассона по переменным $p_{k}, q_{l}$. Она имеет представление

$$
L(w)=\left(\begin{array}{ccccccc}
v_{1} & c_{1} & 0 & \ldots & \ldots & 0 & 0 \\
c_{1} & v_{2} & c_{2} & 0 & \ldots & 0 & 0 \\
0 & c_{2} & v_{3} & \ddots & \ddots & \vdots & \vdots \\
\vdots & 0 & \ddots & \ddots & \ddots & 0 & \vdots \\
\vdots & \vdots & \ddots & \ddots & \ddots & \ddots & 0 \\
0 & 0 & \ldots & 0 & \ddots & \ddots & c_{n-1} \\
w c_{n} & 0 & \ldots & \ldots & 0 & c_{n-1} & v_{n}
\end{array}\right),
$$

где $c_{k}=e^{\left(q_{k}-q_{k+1}\right) / 2}, v_{k}=-p_{k}$. Заметим, что представление Лакса не единственно для открытой цепочки, но унифицирует методы спектральной кривой для случаев открытой и периодической цепочек. Коммутативное семейство определяется коэффициентами характеристического многочлена

$$
\operatorname{det}(L(w)-\lambda)=0
$$

который, в свою очередь, определяет рациональную кривую. Эту кривую можно интерпретировать как предел гиперэллиптической кривой в периодическом случае. Действительно, открытая цепочка является пределом системы в гораздо более общей постановке задачи - для обобщенной системы Тоды.

Наметим основную стратегию. Мы начинаем с ввода производящей функции для классических интегралов общего вида для системы Тоды. Эта функция возникает как предел классического характеристического многочлена для модели Годена [3] с конкретным выбором магнитного члена. Замечая затем, что данное семейство инвариантно относительно действия борелевской группы, мы реализуем редукцию Адлера-Костанта-Саймса (АКС) по отношению к разложению $\mathfrak{g l}_{n}=\mathfrak{b} \oplus \mathfrak{s o}_{n}$. Эту идею можно распространить на квантовый уровень. Мы используем те же элементы: квантовую модель Годена со специальным магнитным членом и ее специальный предел. Мы демонстрируем инвариантность получающегося коммутативного семейства по отношению к действию борелевской группы и реализуем квантовую редукцию $\mathrm{AKC.}$

\section{2. СПЕКТРАЛЬНАЯ КРИВАЯ ДЛЯ КЛАССИЧЕСКОЙ СИСТЕМЫ}

2.1. Определение. Система Тоды общего вида для алгебры Ли $\mathfrak{g l}_{n}$ получается в рамках так называемой процедуры отсечения. Рассмотрим симметричную матрицу $A$, элементы которой представляют собой генераторы борелевской подалгебры $\mathfrak{b}=\mathfrak{b}_{-}:$

$$
A=\sum_{i \leqslant j}\left(E_{i j}+E_{j i}\right) \otimes e_{i j},
$$

где $E_{i j}$ - генераторы алгебры $\operatorname{End}\left(\mathbb{C}^{n}\right), e_{i j}$ при $i \geqslant j-$ генераторы алгебры Ли $\mathfrak{b}$. Матричные коэффициенты интерпретируются как функции на двойственном пространстве к алгебре Ли $\mathfrak{b}^{*}$, которое представляет собой пуассоново пространство со скобкой Кириллова-Костанта. Определим также специальную матрицу $A_{k}(\lambda)$, 
получаемую стиранием $k$ правых колонок и $k$ верхних строк матрицы $A-\lambda \mathrm{Id}$. Coгласно результату работы [1] полный набор корней всех многочленов

$$
\Delta_{k}(\lambda)=\operatorname{det} A_{k}(\lambda)=\sum_{i} I_{k, i} \lambda^{i}
$$

составляет коммутативное семейство. Альтернативный способ введения коммутативного семейства состоит в использовании аффинных координат - отношений коэффициентов многочлена $\Delta_{k}(\lambda)$. Можно использовать тот факт, что $\Delta_{n-k}(\lambda)=$ $\Delta_{n-k}-$ главный по $\lambda$ член в $\Delta_{k}(\lambda)$. Это позволяет ввести семейство характеристических многочленов

$$
P_{k}(\lambda)=\frac{\Delta_{k}(\lambda)}{\Delta_{n-k}(\lambda)}, \quad k=0, \ldots, \frac{n}{2} .
$$

2.2. Производящая функция. Рассмотрим матрицу $A$, соответствующую полной алгебре Ли $\mathfrak{g l}_{n}$ :

$$
A=\sum_{i, j} E_{i j} \otimes e_{i j}
$$

Нам потребуется обозначение

$$
\Omega_{\varepsilon}=\left(\begin{array}{cccc}
0 & \cdots & 0 & 1 \\
\vdots & . \cdot & \varepsilon & 0 \\
0 & \varepsilon^{n-2} & . \cdot & \vdots \\
\varepsilon^{n-1} & 0 & \cdots & 0
\end{array}\right) .
$$

Можно упорядочить коэффициенты миноров (2) в производящий ряд. Рассмотрим характеристический многочлен $P(z, \lambda, \varepsilon)=\operatorname{det}\left(A z^{-1}+\Omega_{\varepsilon}-\lambda \mathrm{Id}\right)$, который представляет собой многочлен по переменным $z^{-1}, \lambda, \varepsilon$. Разложим его в сумму компонент $I_{k}(z, \lambda, \varepsilon)$, однородных степени $k$ по $\left(z^{-1}, \lambda\right)$, каждая из которых представляет собой многочлен по $\varepsilon$ с главным членом $I_{k}^{0}(z, \lambda)$ :

$$
P(z, \lambda, \varepsilon)=\sum_{k} I_{k}(z, \lambda, \varepsilon)=\sum_{k} \varepsilon^{k(k-1) / 2}\left(I_{k}^{0}(z, \lambda)+O(\varepsilon)\right)
$$

Обозначим через $I_{k, i}$ коэффициенты многочлена $I_{k}^{0}(z, \lambda)$ :

$$
I_{k}^{0}(z, \lambda)=\sum_{i=0}^{k} I_{k, i} z^{-i} \lambda^{k-i}
$$

Тогда, в частности, $\Delta_{k}(\lambda)=I_{k}^{0}(1, \lambda)$.

ЗАмЕчАниЕ 1. Эта конструкция показывает, что, например, миноры коммутируют относительно скобки Кириллова-Костанта на $S\left(\mathfrak{g l}_{n}\right)$. Действительно, такая алгебра представляет собой предельный случай коммутативной пуассоновой алгебры, получаемой путем сдвига аргумента, или, эквивалентно, путем рассмотрения соответствующей модели Годена. 
2.3. Схема AKC. Напомним центральную концепцию теории интегрируемых систем - схему АКС. Здесь мы будем использовать следующий вариант: пусть $\mathfrak{g}-$ алгебра Ли, представимая в виде прямой суммы двух подалгебр: $\mathfrak{g}=\mathfrak{g}_{+} \oplus \mathfrak{g}_{-}$. Симметрическая алгебра $S(\mathfrak{g})$ здесь рассматривается как алгебра функций на двойственном пространстве $\mathfrak{g}^{*}$. Имеется естественное отображение проекции

$$
i: S(\mathfrak{g}) \rightarrow S\left(\mathfrak{g}_{+}\right)
$$

связанное с разложением симметрической алгебры

$$
S(\mathfrak{g})=S\left(\mathfrak{g}_{+}\right) \oplus \mathfrak{g}_{-} S(\mathfrak{g}) .
$$

Заметим, что $\mathfrak{g}_{-} S(\mathfrak{g})$ - подалгебра Ли. Отображение $i$ можно интерпретировать как ограничение на пространство $\operatorname{Ann}\left(\mathfrak{g}_{-}\right) \in \mathfrak{g}^{*}$. Несмотря на то что это отображение не является, вообще говоря, пуассоновым, оно сохраняет свойство интегрируемости в следующем смысле.

ЛЕмма 1. Пусть $f, h \in S(\mathfrak{g})$ инвариантны относителъно действия $\mathfrak{g}_{+}$алгебрь Ли и коммутируют относительно скобки Кириллова-Костанта. Тогда их образы $i(f), i(h)$ коммутируют относителъно скобки на $S\left(\mathfrak{g}_{+}\right)$.

ДокАЗАтельство. Рассмотрим элементы $f, h \in S(\mathfrak{g})$ и их разложения в соответствии с формулой (6):

$$
f=f_{+}+f_{-}, \quad g=g_{+}+g_{-} .
$$

Тогда

$$
\left\{f_{-}, g_{-}\right\}=\left\{f-f_{+}, g-g_{+}\right\}=\{f, g\}-\left\{f_{+}, g\right\}-\left\{f, g_{+}\right\}+\left\{f_{+}, g_{+}\right\}=\left\{f_{+}, g_{+}\right\} .
$$

Но обе части принимают значения в различных прямых слагаемых (6), а потому обращаются в нуль. Лемма доказана.

ЗАмЕчАниЕ 2. В данном разделе нам требуется рациональное обобщение этого утверждения, которое получается непосредственно.

2.4. Инвариантность. Требуется показать, что отношения $\Delta_{k}(\lambda) / \Delta_{n-k}$ инвариантны относительно борелевской подгруппы нижнетреугольных матриц $B \subset$ $S L(n)$. Сначала покажем, что действие группы на функции $e_{i j}$ можно выразить в терминах действия на оператор Лакса $A$ :

$$
\operatorname{Ad}_{g}(A):=\sum_{i, j} E_{i, j} \otimes \operatorname{Ad}_{g}\left(e_{i j}\right)=\sum_{i, j} \operatorname{Ad}_{g^{\mathrm{T}}}\left(E_{i j}\right) \otimes e_{i j}=g^{\mathrm{T}} A\left(g^{\mathrm{T}}\right)^{-1} .
$$

Действие группы на коэффициенты характеристического многочлена выражается следующим образом:

$$
\begin{aligned}
\operatorname{det}\left(\operatorname{Ad}_{g^{\mathrm{T}}}(A) z^{-1}+\Omega_{\varepsilon}-\lambda\right) & =\operatorname{det}\left(A z^{-1}+\operatorname{Ad}_{\left(g^{\mathrm{T}}\right)^{-1}}\left(\Omega_{\varepsilon}\right)-\lambda\right)= \\
& =\sum_{k} \varepsilon^{k(k-1) / 2}\left(\operatorname{Ad}_{g}\left(I_{k}^{0}(z, \lambda)\right)+O(\varepsilon)\right) .
\end{aligned}
$$


Рассмотрим элемент борелевской группы $g=e^{t e_{j, i}}=1+t e_{j, i}, j>i$. Его действие на матрицу $\Omega_{\varepsilon}$ можно записать как

$$
\left(g^{\mathrm{T}}\right)^{-1} \Omega_{\varepsilon} g^{\mathrm{T}}=\Omega_{\varepsilon}-t E_{n-i+1, j} \varepsilon^{n-i}+t E_{i, n-j+1} \varepsilon^{j-1}-t^{2} \delta_{j, n-1+1} E_{i, j} \varepsilon^{n-i} .
$$

Эта матрица удовлетворяет следующему свойству: младший по $\varepsilon$ элемент в каждой строке и на каждой линии стоит на антидиагонали. Это подтверждается тем, что самый младший член по $\varepsilon$ в характеристическом многочлене (7) такой же, как и в недеформированном выражении.

Покажем, что картановская подгруппа действует характером. Рассмотрим элемент $g=e^{t e_{i, i}}$ из $B$ и его действие

$$
\begin{aligned}
\left(g^{\mathrm{T}}\right)^{-1} \Omega_{\varepsilon} g^{\mathrm{T}}=\Omega_{\varepsilon} & +\varepsilon^{i-1} E_{i, n-i+1}\left(e^{t}-1\right)+\varepsilon^{n-i} E_{n+1-i, i}\left(e^{t}-1\right)+ \\
& +\varepsilon^{n-i} \delta_{i, n+1-i} E_{i, i}\left(e^{t}-1\right)\left(e^{-t}-1\right) .
\end{aligned}
$$

Данное наблюдение позволяет заключить, что антидиагоналые члены в $\Omega_{\varepsilon}$ умножаются на скаляры, а это следующим образом влияет на асимптотики:

$$
\operatorname{Ad}_{g}\left(\Delta_{k}(\lambda)\right)=\chi_{k}(g)\left(\Delta_{k}(\lambda)\right)
$$

где $\chi_{k}(g)$ - соответствующий характер. Таким образом, мы доказали следующую теорему.

ТЕОРема 1. Отношения $\left(I_{k, i}\right)_{+} / I_{k, n-k}$ порождают коммутативную подалгебpy

$$
\left[\left(I_{k, i}\right)_{+} / I_{k, n-k},\left(I_{m, j}\right)_{+} / I_{m, n-m}\right]=0
$$

в поле частных $\mathcal{F}(S(\mathfrak{b}))$.

ЗАмЕчАниЕ 3. На самом деле в таком контексте имеется более широкая инвариантность. Можно рассмотреть башню параболических подалгебр

$$
\mathfrak{b} \subset \mathfrak{p}_{1} \subset \cdots \subset \mathfrak{p}_{n}=\mathfrak{s l}_{n}
$$

таких, что $\mathfrak{p}_{n}$ порождается $\mathfrak{b}$ и положительными генераторами, соответствующими корням $\alpha_{k}, \ldots, \alpha_{n-k-1}$. Рассмотрим также башню параболических групп

$$
B \subset P_{1} \subset \cdots \subset P_{n}=S L_{n} .
$$

Лемма 2. Верно, что $I_{k, i} / I_{k, j} \in \mathcal{F} S\left(\mathfrak{s l}_{n}\right)^{P_{k}}$.

Этот результат можно найти в работе [4].

ЗАмечАниЕ 4. Такое коммутативное семейство и некоторые обобщения, связанные с геометрией многообразий флагов, обсуждаются в работе [5].

\section{3. КВАНТОВАНИЕ}

Мы строим квантовую модель, рассматривая специальный предел коммутативной алгебры Годена, связанной с 3-точечным случаем, также известным как конструкция сдвига аргумента. Кроме того, мы покажем, что эта подалгебра инвариантна относительно действия $B$ на универсальной обертывающей алгебре $U\left(\mathfrak{s l}_{n}\right)$, и дадим квантовый аналог конструкции АКС, который приводит к построению коммутативной алгебры в $U(\mathfrak{b})$. 
3.1. Некоммутативный определитель. Рассмотрим матрицу $B=\sum_{i, j} E_{i j} \otimes$ $B_{i j}$, элементы которой лежат в некоторой ассоциативной алгебре: $B_{i j} \in \mathfrak{R}$. В данном случае мы будем использовать следующее определение для некоммутативного определителя:

$$
\operatorname{det}(B)=\frac{1}{n !} \sum_{\tau, \sigma \in \Sigma_{n}}(-1)^{\tau \sigma} B_{\tau(1), \sigma(1)} \cdots B_{\tau(n), \sigma(n)} .
$$

Имеется эквивалентное определение. Введем оператор $A_{n}$ антисимметризации в $\left(\mathbb{C}^{n}\right)^{\otimes n}$ :

$$
A_{n} v_{1} \otimes \cdots \otimes v_{n}=\frac{1}{n !} \sum_{\sigma \in S_{n}}(-1)^{\sigma} v_{\sigma(1)} \otimes \cdots \otimes v_{\sigma(n)} .
$$

Приведенное выше определение эквивалентно следующему:

$$
\operatorname{det}(B)=\operatorname{Tr}_{1 \ldots n} A_{n} B_{1} \ldots B_{n},
$$

где $B_{k}$ обозначает оператор в $\operatorname{End}\left(\mathbb{C}^{n}\right)^{\otimes n} \otimes A$, задаваемый формулой

$$
B_{k}=\sum_{i, j} 1 \otimes \cdots \otimes \underbrace{E_{i j}}_{k} \otimes \cdots \otimes 1 \otimes B_{i j}
$$

причем след берется по $\operatorname{End}\left(\mathbb{C}^{n}\right)^{\otimes n}$.

3.2. Квантовая спектральная кривая. Рассмотрим ту же матрицу $A$, что и в классическом случае, но интерпретируем ее элементы как генераторы алгебры $U\left(\mathfrak{g l}_{n}\right)$ :

$$
A=\sum_{i, j} E_{i j} \otimes e_{i j}
$$

Рассмотрим производящий ряд

$$
\begin{aligned}
P_{Q}\left(z, \partial_{z}, \varepsilon\right) & =\operatorname{det}\left(A z^{-1}+\Omega_{\varepsilon}-\partial_{z} \mathrm{Id}\right)= \\
& =\sum_{k} Q I_{k}\left(z, \partial_{z}, \varepsilon\right)=\sum_{k} \varepsilon^{k(k-1) / 2}\left(Q I_{k}^{0}\left(z, \partial_{z}\right)+O(\varepsilon)\right) .
\end{aligned}
$$

Здесь, как и в классическом случае, мы сначала рассматриваем однородное разложение в пространстве дифференциальных многочленов по отношению к градуировке $\operatorname{deg}(z)=-1, \operatorname{deg}\left(\partial_{z}\right)=1^{1)} ; Q I_{k}\left(z, \partial_{z}, \varepsilon\right)$ - компонента в градуировке $k$. Далее рассмотрим главный член $\varepsilon$-разложения для каждой однородной компоненты $Q I_{k}^{0}\left(z, \partial_{z}\right)$. Коммутативная алгебра в $U\left(\mathfrak{g l}_{n}\right)$ порождается коэффициентами выражения

$$
Q I_{k}^{0}\left(z, \partial_{z}\right)=\sum_{l=0}^{k} Q I_{k, i} z^{-i} \partial_{z}^{k-i}
$$

Эти операторы представляют собой высшие члены $\varepsilon$-разложения соответствующих гамильтонианов Годена и потому коммутируют. Общее утверждение для модели Годена доказано в работе [3], случай с магнитным полем проанализирован в работе [6].

ТеОРема 2. Набор $\left\{Q I_{k, i}\right\} \subset U\left(\mathfrak{g l}_{n}\right)$ порождает коммутативную алгебру $\mathcal{H}_{q}$, которая является квантованием пуассон-коммутативной подалгебры в $S\left(\mathfrak{g l}_{n}\right)$, порожденной элементами $I_{k, i}$.

1) Заметим, что градуировка определена корректно вследствие однородности соотношения в алгебре Витта $\partial_{z} z-z \partial_{z}=1$. 
3.3. Квантовая инвариантность. В настоящем разделе мы рассмотрим коммутативную подалгебру в $U(\mathfrak{b})$, являющуюся квантованием подалгебры гамильтонианов для обобщенной цепочки Тоды. Мы будем придерживаться той же стратегии, что и в классическом случае - найдем инварианты относительно действия борелевской подгруппы $B$ на некоторой локализации алгебры $U(\mathfrak{b})$.

Определим задачу разложения в квантовом случае. Рассмотрим разложение алгебры Ли $\mathfrak{s l}_{n}=b \oplus \mathfrak{s o}_{n}$, которое для универсальных обертывающих алгебр превращается в

$$
U\left(\mathfrak{s l}_{n}\right)=U(\mathfrak{b}) \oplus \mathfrak{s o}_{n} U\left(\mathfrak{s l}_{n}\right),
$$

задаваемое с помощью выбора нормального упорядочения. Обозначим через $a_{+}$ проекцию $a \in U\left(\mathfrak{s l}_{n}\right)$ на первое слагаемое.

Установим некоторое специальное свойство инвариантности квантового коммутативного семейства. Действие элемента группы $g \in B$ на $Q I_{k, i}$ можно реализовать в терминах действия на квантовый оператор Лакса, как и в классическом случае:

$$
\operatorname{det}\left(g^{\mathrm{T}} A g^{\mathrm{T}^{-1}} z^{-1}+\Omega_{\varepsilon}-\partial_{z}\right)=\operatorname{det}\left(A z^{-1}+g^{\mathrm{T}^{-1}} \Omega_{\varepsilon} g^{\mathrm{T}}-\partial_{z}\right),
$$

поэтому вопрос сводится к свойствам матрицы $\Omega_{\varepsilon}$. Для доказательства равенства (10) нам потребуется следующая лемма.

Лемма 3. Пусть $g \in G L_{n}$, a $L$ - элемент из $\mathrm{Mat}_{n} \otimes \Re$ для некоторой ассоииативной алгебры $\mathfrak{R}$. Тогда $\operatorname{det}\left(g L g^{-1}\right)=\operatorname{det}(L)$.

ДокАЗАТЕЛьство. Воспользуемся альтернативной формулой для некоммутативного определителя

$$
\operatorname{det}(L)=\operatorname{Tr}_{1, \ldots, n} A_{n} L_{1} L_{2} \ldots L_{n}
$$

Тогда

$$
\begin{aligned}
\operatorname{det}\left(g L g^{-1}\right) & =\operatorname{Tr}_{1, \ldots, n} A_{n} g_{1} L_{1} g_{1}^{-1} g_{2} L_{2} g_{2}^{-1} \ldots g_{n} L_{n} g_{n}^{-1}= \\
& =\operatorname{Tr}_{1, \ldots, n} A_{n} g_{1} g_{2} \ldots g_{n} L_{1} L_{2} \ldots L_{n} g_{1}^{-1} g_{2}^{-1} \ldots g_{n}^{-1}= \\
& =\operatorname{Tr}_{1, \ldots, n} g_{1}^{-1} g_{2}^{-1} \ldots g_{n}^{-1} A_{n} g_{1} g_{2} \ldots g_{n} L_{1} L_{2} \ldots L_{n}= \\
& =\operatorname{Tr}_{1, \ldots, n} A_{n} L_{1} L_{2} \ldots L_{n}=\operatorname{det}(L) .
\end{aligned}
$$

Здесь мы воспользовались тем, что $\operatorname{Tr}(A B)=\operatorname{Tr}(B A)$ для матриц с коммутирующими элементами $\left[A_{i j}, B_{k l}\right]=0$, и тем фактом, что на тензорном произведении векторных представлений действие групповой алгебры симметрической группы коммутирует с диагональным действием линейной группы. Лемма доказана.

3.4. Квантовая лемма АКС. По аналогии с классическим случаем мы показали, что группа $B$ действует на $Q I_{k}^{0}\left(z, \partial_{z}\right)$ характером $\chi_{k}(b)$, где $b \in B$. Обозначим той же буквой характер на алгебре Ли $\chi_{k}(X)$ при $X \in \mathfrak{b}$, так что

$$
\operatorname{ad}_{X} Q I_{k, i}=\chi_{k}(X) Q I_{k, i} \text {. }
$$

Введем также обозначение $\eta_{k}(m)$ с помощью формулы

$$
m a=a \eta_{k}(m),
$$

где $a=Q I_{k, i}$ и $m \in U(\mathfrak{b})$. Если $m$ - моном $m=b_{1} \ldots b_{s}$, то

$$
\eta_{k}(m)=\left(b_{1}+\chi_{k}\left(b_{1}\right)\right) \ldots\left(b_{s}+\chi_{k}\left(b_{s}\right)\right) .
$$


ЛЕмма 4. Рассмотрим разложения типа (9) двух элементов нашей коммутативной алгебры:

$$
Q I_{k, i}=a=a_{+}+a_{-}, \quad Q I_{l, j}=b=b_{+}+b_{-} .
$$

Тогда $a_{+} \eta_{k}\left(b_{+}\right)=b_{+} \eta_{l}\left(a_{+}\right)$.

ЗАмЕЧАНИЕ 5. Лемма 4 представляет собой аналог леммы АКС.

ДокАЗАТЕЛьство. Воспользуемся условием коммутативности

$$
\begin{aligned}
{\left[a_{-}, b_{-}\right]=} & {\left[a-a_{+}, b-b_{+}\right]=[a, b]+\left[a_{+}, b_{+}\right]-\left[a, b_{+}\right]-\left[a_{+}, b\right]=} \\
= & {[a, b]+\left[a_{+}, b_{+}\right]-a b_{+}+a \eta_{k}\left(b_{+}\right)-b \eta_{l}\left(a_{+}\right)+b a_{+}=} \\
= & {\left[a_{+}, b_{+}\right]-a_{-} b_{+}-a_{+} b_{+}+a_{-} \eta_{k}\left(b_{+}\right)+a_{+} \eta_{k}\left(b_{+}\right)-} \\
& \quad-b_{-} \eta_{l}\left(a_{+}\right)-b_{+} \eta_{l}\left(a_{+}\right)+b_{+} a_{+}+b_{-} a_{+} .
\end{aligned}
$$

Положительная часть выражения (13) равна

$$
0=a_{+} \eta_{k}\left(b_{+}\right)-b_{+} \eta_{l}\left(a_{+}\right)
$$

Лемма доказана.

Мы хотели бы рассмотреть локализацию алгебры $U(\mathfrak{b})$ с мультипликативным множеством $S$, порожденным $\left\{Q I_{k, n-k}\right\}$ - набором старших членов всех частичных квантовых характеристических многочленов.

ЛЕмма 5. $S$ является правым множеством Оре.

Напомним условия Оре. $S \subset A$ представляет собой правое множество Оре, если

1) для всех $s \in S$ и $a \in A$ существуют $s^{\prime} \in S$ и $a^{\prime} \in A$ такие, что $s a^{\prime}=a s^{\prime}$;

2) для всех $a_{1}, a_{2} \in A$ и $s \in S:\left(s a_{1}=s a_{2}\right) \Rightarrow\left(\exists s^{\prime} \in S: a_{1} s^{\prime}=a_{2} s^{\prime}\right)$.

Второе условие тривиально, поскольку алгебра $U\left(\mathfrak{s l}_{n}\right)$ не имеет делителей нуля. Первое условие выполняется в силу (12).

Теорема 3. Рассмотрим локализацию $\operatorname{loc}_{S} U(\mathfrak{b})$. Отношения $\left(Q I_{k, i}\right)_{+} / Q I_{k, n-k}$ порождают коммутативную подалгебру:

$$
\left[\left(Q I_{k, i}\right)_{+} / Q I_{k, n-k},\left(Q I_{m, j}\right)_{+} / Q I_{m, n-m}\right]=0
$$

которая представляет собой квантование классической подалгебры интегралов обобщенной системы Тодъ.

ДокАЗАТЕЛЬСтво. Воспользуемся обозначениями

$$
a=Q I_{k, i}, \quad b=Q I_{m, j}, \quad c=Q I_{k, n-k}, \quad d=Q I_{m, n-m} .
$$

Тогда

$$
a_{+} c^{-1} b_{+} d^{-1}-b_{+} d^{-1} a_{+} c^{-1}=a_{+} \eta_{k}\left(b_{+}\right) c^{-1} d^{-1}-b_{+} \eta_{m}\left(a_{+}\right) d^{-1} c^{-1}
$$

Это равно нулю вследствие леммы 4 и коммутативности $d$ и $c$ в обеих алгебрах. 


\section{4. КОММЕНТАРИИ}

Застава Дринфельда [7] является некоторым пополнением пространства отображений фиксированной степени из $\mathbb{C} P^{1}$ до многообразия флагов $\mathcal{F}_{n}$ :

$$
\mathcal{M}_{\bar{d}}=\left\{f: \mathbb{C} P^{1} \rightarrow \mathcal{F}_{n}, c_{1}\left(f^{*} \mathcal{L}_{i}\right)=d_{i} \in H^{1}\left(\mathbb{C} P^{1}\right)\right\},
$$

где $\mathcal{L}_{i}$ - ансамбль канонических линейных расслоений на многообразии флагов, $\mathcal{L}_{i}=V_{i}^{\wedge i}$.

С другой стороны, можно рассмотреть пространство рациональных отображений

$$
L_{d}=\left\{F: \mathbb{C} P^{1} \rightarrow \operatorname{Mat}_{n}\right\},
$$

допускающих проекцию на пространство отображений в многообразие флагов $\mathcal{M}_{\bar{d}}$ при $\bar{d}=(-d,-2 d, \ldots,-n d)$. Тогда, используя вариант гамильтоновой редукции, можно построить коммутативное семейство в $C\left(\mathcal{T}^{*} L_{d}\right)$ аналогично тому, как это делается для случая обобщенной системы Тоды. Мы полагаем, что такое построение тесно связано с потоками Гельфанда-Цетлина на многообразии флагов, которое сохраняет свое значение на квантовом уровне [7].

Благодарности. Автор выражает благодарность своим коллегам Ю. Чернякову и Г. Шарыгину за плодотворные обсуждения рассматриваемой проблемы. Работа выполнена при частичной финансовой поддержке Simons Foundation, РФФИ (грант № 11-01-00197), Программы поддержки ведущих научных школ (грант НШ-5413. 2010.1), РФФИ-Консорциума E.I.N.S.T.E.I.N (грант № 09-01-92437), РФФИ-CNRS (грант № 11-01-93105-НЦНИЛ_а), а также Министерства образования и науки РФ в рамках контракта № 14.740 .11 .0081 .

\section{Список литературы}

[1] P. Deift, L. C. Li, T. Nanda, C. Tomei, Commun. Pure Appl. Math., 39:2 (1986), 183-232.

[2] I. Krichever, K. L. Vaninsky, "The periodic and open Toda lattice", Mirror symmetry IV (Montréal, Canada, 2000), AMS/IP Stud. Adv. Math., 33, eds. E. D'Hoker et al., AMS, Providence, RI, 2002, 139-158, arXiv: hep-th/0010184.

[3] Д. В. Талалаев, Функи. анализ и его прил., 40:1 (2006), 86-91.

[4] M. Gekhtman, M. Shapiro, Commun. Pure Appl. Math., 52:1 (1999), 53-84, arXiv: solv-int/9704011.

[5] Yu. Chernyakov, A. Sorin, Involutive families of integrals of the full symmetric Toda system on a generic orbit, preprint (to appear).

[6] A. Chervov, D. Talalaev, Quantum spectral curves, quantum integrable systems and the geometric Langlands correspondence, arXiv: hep-th/0604128.

[7] M. Finkelberg, L. Rybnikov, Quantization of Drinfeld Zastava, arXiv: 1009.0676. 